\title{
The Association of Pre-Kidney Transplant Dialysis Modality with de novo Posttransplant Heart Failure
}

\author{
Colin R. Lenihan ${ }^{a}$ Sai Liu ${ }^{a} \quad$ Medha Airy ${ }^{b} \quad$ Carl Walther ${ }^{b} \quad$ Maria E. Montez-Rath ${ }^{a}$ \\ Wolfgang C. Winkelmayer ${ }^{\mathrm{b}}$ \\ aDivision of Nephrology, Stanford University School of Medicine, Palo Alto, CA, USA; bSelzman Institute for \\ Kidney Health, Section of Nephrology, Baylor College of Medicine, Houston, TX, USA
}

\section{Keywords}

Heart failure - End-stage renal disease · Hemodialysis .

Peritoneal dialysis - Kidney transplantation - Outcomes .

Cohort study · US Renal Data System

\begin{abstract}
Background: Heart failure (HF) after kidney transplantation is a significant but understudied problem. Pretransplant dialysis modality could influence incident HF risk through differing cardiac stressors. However, whether pretransplant dialysis modality is associated with the development of posttransplant HF is unknown. Methods: We used the US Renal Data System to assemble a cohort of 27,701 patients who underwent their first kidney transplant in the USA between the years 2005 and 2012 and who had Medicare fee-for-service coverage for $>6$ months preceding their transplant date. Patients with any HF diagnosis prior to transplant were excluded. Detailed baseline patient characteristics and comorbidities were abstracted. The outcome of interest was de novo posttransplant HF. Pretransplant dialysis modality was defined as the dialysis modality used at the time of transplant. We conducted time-to-event analyses using Cox regression. Death was treated as a competing risk in the
\end{abstract}

karger@karger.com www.karger.com/crm

Karger $\stackrel{\text { ' }}{5}$
(C) 2021 The Author(s)

Published by S. Karger AG, Basel

This is an Open Access article licensed under the Creative Commons Attribution-NonCommercial-4.0 International License (CC BY-NC) (http://www.karger.com/Services/OpenAccessLicense), applicable to the online version of the article only. Usage and distribution for commercial purposes requires written permission. study's primary analysis. Graft failure was included as a timevarying covariate. Results: Among eligible patients, $81 \%$ were treated with hemodialysis prior to transplant, and hemodialysis patients were more likely to be male, had a shorter dialysis vintage, and had more diabetes and vascular disease diagnoses. When adjusted for all available demographic and clinical data, pretransplant treatment with hemodialysis (vs. peritoneal dialysis) was associated with a $19 \%$ increased risk in de novo posttransplant HF, with subdistribution HR 1.19 (95\% Cl: 1.09-1.29). Conclusions: Our results suggest that choice of pretransplant dialysis modality may impact the development of posttransplant HF.

\footnotetext{
(c) 2021 The Author(s)

Published by S. Karger AG, Basel
}

\section{Introduction}

For many patients with end-stage kidney disease (ESKD), kidney transplantation offers the best outcome in terms of survival and quality of life $[1,2]$. However, survival of patients with a kidney transplant is still reduced compared to that of the general population, an effect attributable, at least in part, to an excess risk of cardiovascular disease in the kidney transplant population 
[3]. Heart failure (HF) following kidney transplant is a particularly important problem. Posttransplant HF is the most frequent cardiovascular cause for hospital admission in the 2 years following transplant and is associated with reduced patient and graft survival $[4,5]$.

Patients with ESKD are exposed to a myriad of both traditional and nontraditional risk factors for HF [6-12]. Chronic intermittent hemodialysis (vs. peritoneal dialysis) exposes patients to a number of unique cardiac stressors including (1) frequent and rapid intravascular volume shifts, (2) myocardial stunning, and (3) the presence of AV shunts that usually remain in situ after they receive a kidney transplant. We therefore hypothesized that those patients undergoing hemodialysis (vs. peritoneal dialysis) would be at higher risk for posttransplant HF. Herein, we formally examine the association between pretransplant dialysis modality and the incidence of de novo posttransplant HF using a large, population-based US ESKD registry.

\section{Methods}

\section{Study Cohort}

We used the US Renal Data System (USRDS) dataset to identify adult patients who underwent their first kidney transplant in the USA between January 1, 2005, and September 30, 2012. We required that patients have at least 6 months of Medicare Parts A and $\mathrm{B}$ coverage prior to their kidney transplant. Prior diagnoses of HF were identified using International Classification of Diseases, 9th revision (ICD-9), codes of 428.xx, 402.01, 402.11, 402.91, 404.01, 404.03, 404.11, 404.13, 404.91, and 404.93. We excluded patients with any inpatient or outpatient HF claims in up to 2 years prior to kidney transplant. We additionally excluded (1) patients for whom data on pretransplant dialysis modality were missing, (2) patients that received a simultaneous kidney-pancreas transplant, and (3) patients for whom there were no Medicare claims visible in the 2 years prior to transplant.

\section{Exposure}

The exposure of interest was the last pretransplant dialysis modality (hemodialysis vs. peritoneal dialysis) as reported in the USRDS treatment history files.

\section{Outcome}

The outcome was de novo posttransplant HF. HF was identified using ICD-9 claims. To ascertain de novo HF, we required either 1 posttransplant inpatient HF claim or 1 outpatient HF claim followed by another either inpatient or outpatient HF claim within 30 days of the first. For outpatient HF diagnosis, the date of the first outpatient claim was the date used for de novo HF. Patients were censored at the end of the study (September 30, 2015), loss of Medicare Parts A and B coverage, or at 3 years after transplant (as many patients lose Medicare coverage at this point).

\section{Patient Characteristics}

For each patient, we abstracted demographic (age at time of transplant, sex, and race [white, black, or others]), dialysis (cause of ESKD [diabetes, hypertension, glomerulonephritis, or others], total dialysis vintage, duration of last pretransplant dialysis modality, and BMI), and transplant (blood type, calculated panel-reactive antibody, donor-recipient human leukocyte antigen match, cold ischemia time, donor age, donor sex, donor type, and any history of prior nonkidney solid organ transplant) variables. We also identified a wide array of pretransplant comorbidities using ICD-9 codes in the claims-based algorithm which required the presence of either 1 inpatient or 2 outpatient claims (not on the same day). The pretransplant comorbidities abstracted were alcohol dependence, arrhythmia, cancer, cerebrovascular disease, chronic pulmonary disease, coronary artery disease, diabetes, hypertension, liver disease, peripheral arterial disease, tobacco use, and valvular heart disease (online suppl. Table 1; for all online suppl. material, see www.karger.com/doi/10.1159/000518535).

We also abstracted information regarding pretransplant skilled nursing facility admissions, inpatient hospital days, and non-nephrology outpatient visits in the 6 months prior to transplant. Graft failure, defined as the need for dialysis or re-transplant, was identified from the USRDS patient file and was treated as a timevarying covariate.

\section{Statistical Analysis}

Baseline characteristics were tabulated for all patients as well as separately for the pretransplant hemodialysis and peritoneal dialysis groups. Continuous variables were presented as either means with standard deviations or medians with interquartile ranges where appropriate. Categorical variables were expressed as percentages. We presented cumulative incidence function plots to compare 3-year cumulative incidence of HF and death by pretransplant modality type.

We estimated unadjusted and incrementally adjusted sub-distribution or cause-specific hazard ratios for de novo posttransplant HF by pretransplant dialysis modality (with peritoneal dialysis being the reference). All models were stratified by the era of transplant; 2005-06, 2007-08, 2009-10, and 2011-12. Models 1-4 were incrementally adjusted as follows: model 1 - time-varying graft failure; model 2 - model 1 plus age at time of transplant, sex, race, $\mathrm{BMI}$, cause of ESRD, dialysis vintage, and duration of last pretransplant dialysis modality; model 3 - model 2 plus comorbidities, health care utilization metrics (nursing home stay, number of hospital days, and number of non-nephrology clinic visits), and prior solid organ transplant status and; model 4 - model 3 plus transplant characteristics. The primary analysis treated death as a competing risk and generated sub-distribution hazard ratios using the Kaplan-Meier multiple imputation method $[13,14]$. A secondary analysis treated death as a censoring event. Both analyses used extended Cox models. We tested for proportional hazards by looking at the correlation of the scaled Schoenfeld residuals with time and found no evidence that the log-hazard ratio changed with followup time for any of the covariates that were included in the model. The $p$ value for the global test was 0.49 . We additionally tested a number of pretransplant patient characteristics as effect modifiers of pretransplant dialysis modality (hemodialysis vs. peritoneal dialysis) on de novo posttransplant HF in the primary analysis. The pretransplant characteristics that were tested were (1) age at time of transplant, (2) race, (3) dialysis vintage, (4) dialysis modality 


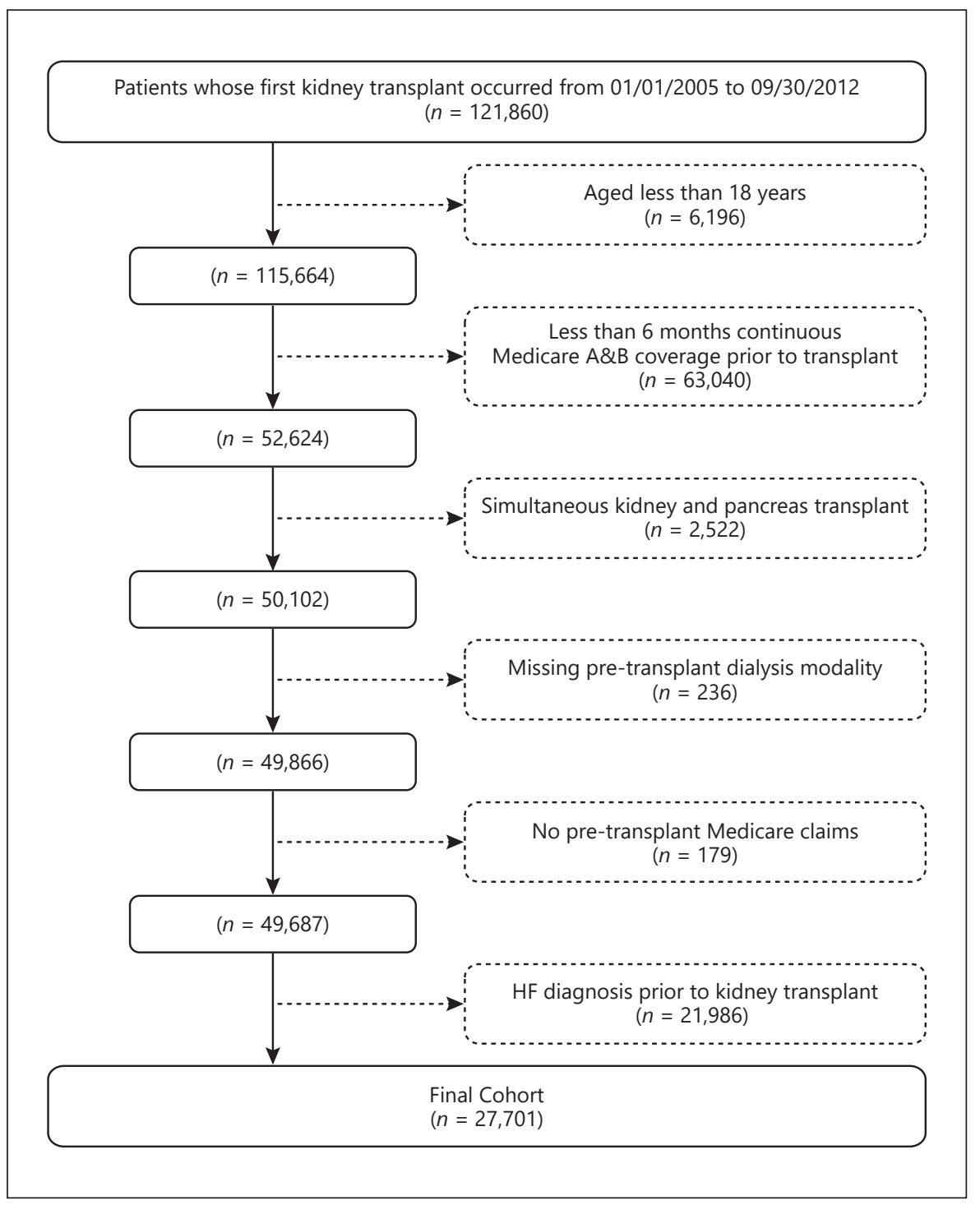

Fig. 1. Cohort flowchart. HF, heart failure.

vintage, (5) BMI, (6) the presence of coronary artery disease, and (7) the presence of diabetes mellitus.

In our cohort of 27,701 patients, $6,842(24.7 \%)$ had at least 1 variable missing. The variables most frequently missing were calculated panel-reactive antibody (15\%) and cold ischemia time (8\%). Data were assumed to be missing at random. Missing data were handled using multiple imputation by fully conditional specification as implemented in SAS, and 25 imputed datasets were obtained for the primary outcome. In addition to the exposure and all covariates included in the analysis model, the imputation model also included the event indicator and the Nelson-Aalen estimator of the cumulative marginal hazard. Imputation models were run separately for the main analysis (to calculate sub-distribution HR). Imputation models were stratified by treatment modality. Statistical analyses were performed using SAS software, version 9.4 (SAS Institute, Inc., Cary, NC, USA), R version 3.1.2, and Stata MP, version 13.1 (StataCorp, College Station, TX, USA).

Dialysis Modality and Posttransplant Heart Failure

\section{Results}

A total of 27,701 adults who underwent their first kidney transplant and satisfied all inclusion criteria were included in the study (Fig. 1). Of these, $81 \%$ of patients were treated with hemodialysis prior to transplant while the remaining $19 \%$ were treated with peritoneal dialysis for a median of 3.2 and 3.7 years, respectively. The baseline characteristics of the study cohort stratified by pretransplant dialysis modality are shown in Table 1. Patients treated with hemodialysis prior to transplant were more likely to be male, African American, had a shorter dialysis vintage, and had a greater burden of diabetes and vascular disease. 
Table 1. Baseline characteristics of US patients who underwent their first kidney transplant between 2005 and 2012, altogether and stratified by pretransplant dialysis modality

\begin{tabular}{|c|c|c|c|}
\hline & $\begin{array}{l}\text { All } \\
(N=27,701)\end{array}$ & $\begin{array}{l}\mathrm{HD} \\
(N=22,375)\end{array}$ & $\begin{array}{l}\mathrm{PD} \\
(N=5,326)\end{array}$ \\
\hline \multicolumn{4}{|l|}{ Baseline characteristics } \\
\hline Female, $n(\%)$ & $10,878(39.3)$ & $8,268(37.0)$ & $2,610(49.0)$ \\
\hline \multicolumn{4}{|l|}{ Age, years } \\
\hline Mean (SD) & $47(14)$ & $47(14)$ & $46(15)$ \\
\hline Median (IQR) & $48(36,58)$ & $48(37,58)$ & $47(35,58)$ \\
\hline \multicolumn{4}{|l|}{ Race, $n(\%)$} \\
\hline White & $16,313(58.9)$ & $12,760(57.0)$ & $3,553(66.7)$ \\
\hline Black & $8,909(32.2)$ & $7,692(34.4)$ & $1,217(22.9)$ \\
\hline Others & $2,440(8.8)$ & $1,891(8.5)$ & $549(10.3)$ \\
\hline \multicolumn{4}{|l|}{ Cause of ESKD, $n(\%)$} \\
\hline Diabetes & $6,991(25.2)$ & $6,042(27.0)$ & $949(17.8)$ \\
\hline Hypertension & $7,187(25.9)$ & $5,439(24.3)$ & $1,748(32.8)$ \\
\hline Glomerulonephritis & $6,905(24.9)$ & $5,704(25.5)$ & $1,201(22.5)$ \\
\hline Others & $6,519(23.5)$ & $5,111(22.8)$ & $1,408(26.4)$ \\
\hline \multicolumn{4}{|l|}{ Year of ESKD, $n(\%)$} \\
\hline 2005-2006 & $7,199(26.0)$ & $5,801(25.9)$ & $1,398(26.2)$ \\
\hline 2007-2008 & $7,068(25.5)$ & $5,809(26.0)$ & $1,259(23.6)$ \\
\hline $2009-2010$ & $7,130(25.7)$ & $5,754(25.7)$ & $1,376(25.8)$ \\
\hline 2011-2012 & $6,304(22.8)$ & $5,011(22.4)$ & $1,293(24.3)$ \\
\hline \multicolumn{4}{|l|}{$\mathrm{BMI}$ at transplant, $\mathrm{kg} / \mathrm{m}^{2}$} \\
\hline Mean (SD) & $27.9(5.2)$ & $27.6(5.0)$ & $28.0(5.2)$ \\
\hline Median (IQR) & $27.6(24.0,31.6)$ & $27.6(24.0,31.7)$ & $27.3(23.8,31.1)$ \\
\hline$<18.5, n(\%)$ & $530(1.9)$ & $399(1.8)$ & $131(2.5)$ \\
\hline $18.5-24.9, n(\%)$ & $8,164(29.5)$ & $6,560(29.3)$ & $1,604(30.1)$ \\
\hline $25-29.9, n(\%)$ & $9,370(33.8)$ & $7,498(33.5)$ & $1,872(35.1)$ \\
\hline$\geq 30, n(\%)$ & $9,172(33.1)$ & $7,517(33.6)$ & $1,655(31.1)$ \\
\hline \multicolumn{4}{|c|}{ Dialysis vintage (time since initiation of dialysis, years) } \\
\hline Mean (SD) & $4.0(2.7)$ & $3.5(2.3)$ & $4.1(2.7)$ \\
\hline Median (IQR) & $3.6(2.1,5.2)$ & $3.2(1.9,4.6)$ & $3.7(2.2,5.3)$ \\
\hline$<2.5, n(\%)$ & $8,416(30.4)$ & $6,546(29.3)$ & $1,870(35.1)$ \\
\hline $2.5-5, n(\%)$ & $11,755(42.4)$ & $9,384(41.9)$ & $2,371(44.5)$ \\
\hline $5-9, n(\%)$ & $6,308(22.8)$ & $5,347(23.9)$ & $961(18.0)$ \\
\hline$\geq 9, n(\%)$ & $1,222(4.4)$ & $1,098(4.9)$ & $124(2.3)$ \\
\hline \multicolumn{4}{|c|}{ Duration of last dialysis modality, years } \\
\hline Mean (SD) & $3.5(2.5)$ & $2.7(1.9)$ & $3.7(2.5)$ \\
\hline Median (IQR) & $3.2(1.7,4.7)$ & $2.4(1.2,3.7)$ & $3.4(1.8,4.9)$ \\
\hline$<2.5, n(\%)$ & $10,729(38.7)$ & $7,967(35.6)$ & $2,762(51.9)$ \\
\hline $2.5-5, n(\%)$ & $10,929(39.5)$ & $8,985(40.2)$ & $1,944(36.5)$ \\
\hline $5-9, n(\%)$ & $5,196(18.8)$ & $4,619(20.6)$ & $577(10.8)$ \\
\hline$\geq 9, n(\%)$ & $847(3.1)$ & $804(3.6)$ & $43(0.8)$ \\
\hline \multicolumn{4}{|l|}{ Comorbidities, $n(\%)$} \\
\hline Diabetes mellitus & $11,229(40.5)$ & $9,624(43.0)$ & $1,605(30.1)$ \\
\hline Alcohol dependence & $451(1.6)$ & $400(1.8)$ & $51(1.0)$ \\
\hline CAD & $6,835(24.7)$ & $5,779(25.8)$ & $1,056(19.8)$ \\
\hline COPD & $3,940(14.2)$ & $3,226(14.4)$ & $714(13.4)$ \\
\hline CVD & $1,903(6.9)$ & $1,623(7.3)$ & $280(5.3)$ \\
\hline Cerebral bleed & $256(0.9)$ & $222(1.0)$ & $34(0.6)$ \\
\hline Cancer & $1,839(6.6)$ & $1,543(6.9)$ & $296(5.6)$ \\
\hline Hypertension & $25,536(92.2)$ & $20,797(92.9)$ & $4,739(89.0)$ \\
\hline VHD & $3,388(12.2)$ & $2,890(12.9)$ & $498(9.4)$ \\
\hline PVD & $5,233(18.9)$ & $4,731(21.1)$ & $502(9.4)$ \\
\hline Liver disease & $4,496(16.2)$ & $3,834(17.1)$ & $662(12.4)$ \\
\hline Tobacco use & $2,397(8.7)$ & $2,010(9.0)$ & $387(7.3)$ \\
\hline Arrhythmia & $1,463(5.3)$ & $1,252(5.6)$ & $211(4.0)$ \\
\hline
\end{tabular}


Table 1 (continued)

\begin{tabular}{|c|c|c|c|}
\hline & $\begin{array}{l}\text { All } \\
(N=27,701)\end{array}$ & $\begin{array}{l}\text { HD } \\
(N=22,375)\end{array}$ & $\begin{array}{l}\mathrm{PD} \\
(N=5,326)\end{array}$ \\
\hline Previous solid organ transplant, $n(\%)$ & $527(1.9)$ & $472(2.1)$ & $55(1.0)$ \\
\hline \multicolumn{4}{|l|}{ Patient blood type, $n(\%)$} \\
\hline $\mathrm{O}$ & $13,249(47.8)$ & $10,706(47.8)$ & $2,543(47.7)$ \\
\hline A & $9,158(33.1)$ & $7,360(32.9)$ & $1,798(33.8)$ \\
\hline $\mathrm{B}$ & $3,965(14.3)$ & $3,225(14.4)$ & $740(13.9)$ \\
\hline $\mathrm{AB}$ & $1,116(4.0)$ & $900(4.0)$ & $216(4.1)$ \\
\hline \multicolumn{4}{|l|}{ Donor type, $n(\%)$} \\
\hline Living & $5,881(21.2)$ & $4,644(20.8)$ & $1,237(23.2)$ \\
\hline Deceased & $17,779(64.2)$ & $14,357(64.2)$ & $3,422(64.3)$ \\
\hline Expanded criteria & $3,868(14.0)$ & $3,225(14.4)$ & $643(12.1)$ \\
\hline \multicolumn{4}{|l|}{ Donor characteristics } \\
\hline \multicolumn{4}{|l|}{ Age, years } \\
\hline Mean (SD) & $39(16)$ & $39(16)$ & $38(16)$ \\
\hline Median (IQR) & $41(27,51)$ & $41.0(27,51)$ & $40.0(26,51)$ \\
\hline Female donor, $n(\%)$ & $12,290(44.4)$ & $9,882(44.2)$ & $2,408(45.2)$ \\
\hline \multicolumn{4}{|l|}{ HLA mismatch, $n(\%)$} \\
\hline 0 & $2,099(7.6)$ & $1,680(7.5)$ & $419(7.9)$ \\
\hline $1-3$ & $6,413(23.2)$ & $5,111(22.8)$ & $1,302(24.4)$ \\
\hline $4-6$ & $18,668(67.4)$ & $15,137(67.7)$ & $3,531(66.3)$ \\
\hline Panel-reactive antibody titer, mean (SD) & $14.7(26.8)$ & $14.5(26.7)$ & $15.3(27.3)$ \\
\hline Median (IQR) & $0.0(0.0,14.0)$ & $0.0(0.0,14.0)$ & $0.0(0.0,16.0)$ \\
\hline Cold ischemia time, mean (SD), h & $15.0(10.6)$ & $15.2(10.6)$ & $14.3(10.3)$ \\
\hline Median (IQR) & $14.5(7.5,21.0)$ & $14.8(8.0,21.1)$ & $14.0(6.1,21.0)$ \\
\hline Nursing home stay, $n(\%)$ & $205(0.7)$ & $185(0.8)$ & $20(0.4)$ \\
\hline Hospital days, mean (SD) & $3(4)$ & $3(5)$ & $3(4)$ \\
\hline Hospital days, median (IQR) & $1(1,2)$ & $1(1,2)$ & $1(1,2)$ \\
\hline Non-nephrology clinic visits, mean (SD) & $13(12)$ & $13(12)$ & $12(12)$ \\
\hline Non-nephrology clinic visits, median (IQR) & $10(5,18)$ & $10(5,18)$ & $9(5,17)$ \\
\hline
\end{tabular}

Several variables had incomplete data; specifically, the proportion of observations missing in the following variables were sex $(<0.1 \%)$, race $(0.1)$, cause of ESKD $(0.4)$, BMI $(1.7 \%)$, blood type $(0.8 \%)$, donor type $(0.6 \%)$, donor age $(0.8 \%)$, donor sex $(0.8 \%)$, HLA mismatch $(1.9 \%)$, calculated panel-reactive antibody titer $(15.5 \%)$, and cold ischemia time (8.5\%). PD, peritoneal dialysis; HD, hemodialysis; IQR, interquartile range; SD, standard deviation; ESKD, end-stage kidney disease; CVD, cerebrovascular disease; CAD, coronary artery disease; COPD, chronic obstructive pulmonary disease; PAD, peripheral arterial disease; VHD, valvular heart disease; HLA, human leukocyte antigen.

Over a mean follow-up of 2.33 years, 3,283 patients $(11.9 \%)$ were diagnosed with de novo HF, with a median time from transplant surgery to a de novo HF diagnosis of 9.7 months (interquartile range, 2.13-22.03); among those previously treated with hemodialysis, $2,809(12.6 \%)$ developed de novo HF compared with $8.8 \%$ of those previously treated with $\mathrm{PD}$.

Pretransplant hemodialysis (vs. peritoneal dialysis) treatment was associated with an increased risk of posttransplant HF (Fig. 2; Table 2). The unadjusted and model 4 -adjusted sub-distribution HRs for de novo HF for hemodialysis versus peritoneal dialysis were 1.36 (95\% CI: $1.27,1.46)$ and 1.19 (95\% CI: 1.09-1.28), respectively.
Results were similar when death was treated as a censoring event. Complete results for both models that use death as a competing risk and death-censoring models are shown in Table 2.

There was a significant interaction identified for BMI $(p$ value for interaction $=0.03)$ and diabetes mellitus $(p$ value for interaction $=0.008$ ), where higher $\mathrm{BMI}$ and the presence of pretransplant diabetes mellitus were synergistically associated with an increased risk of de novo posttransplant HF in those patients treated before transplant with hemodialysis (vs. peritoneal dialysis). More specifically, in patients with a pretransplant BMI of $\geq 30$ $\mathrm{kg} / \mathrm{m}^{2}$, hemodialysis (vs. peritoneal dialysis) treatment 


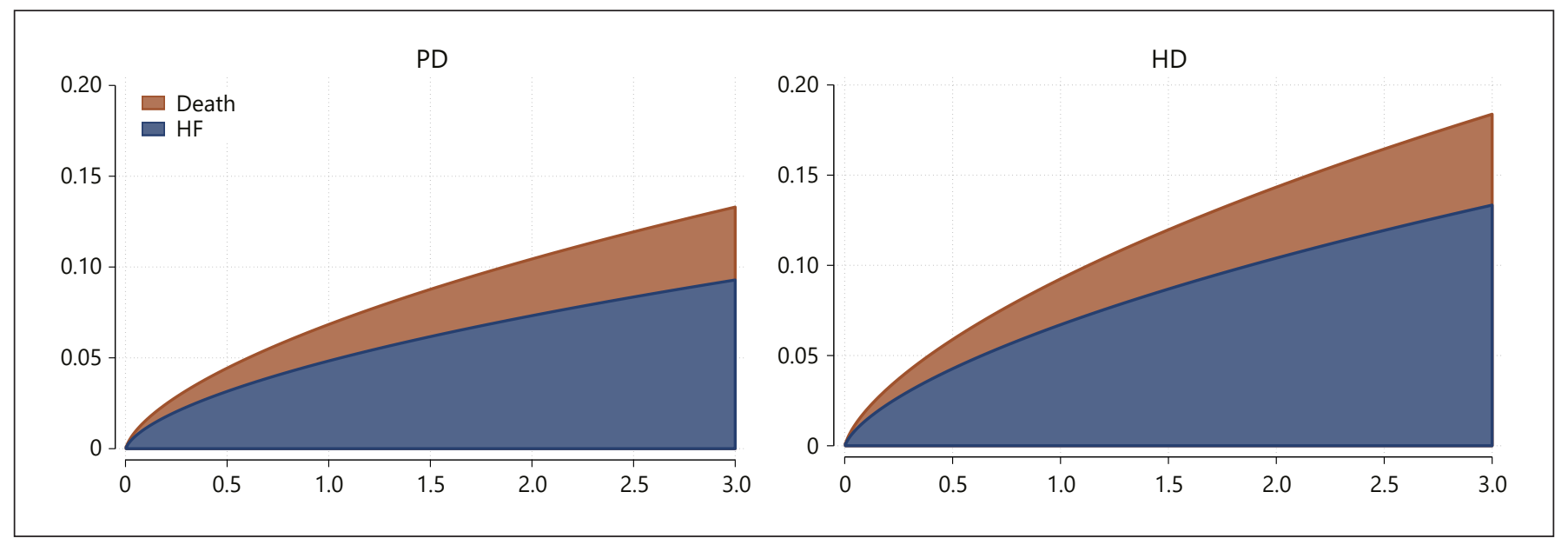

Fig. 2. Three-year cumulative incidence of posttransplant HF and death in patients treated before transplant with $\mathrm{PD}$ and $\mathrm{HD}$. PD, peritoneal dialysis; HD, hemodialysis; HF, heart failure.

Table 2. Risk of de novo posttransplant heart failure in patients treated before transplant with hemodialysis versus peritoneal dialysis

\begin{tabular}{lll}
\hline Models & $\begin{array}{l}\text { Sub-distribution } \\
\text { HR }(95 \% \text { CI })^{*}\end{array}$ & $\begin{array}{l}\text { Cause-specific } \\
\text { HR }(95 \% \text { CI)* }\end{array}$ \\
\hline Model 1 & $1.36(1.27-1.46)$ & $1.37(1.28-1.46)$ \\
Model 2 & $1.24(1.14-1.34)$ & $1.25(1.15-1.34)$ \\
Model 3 & $1.18(1.09-1.28)$ & $1.19(1.09-1.29)$ \\
Model 4 & $1.19(1.09-1.28)$ & $1.20(1.10-1.29)$ \\
\hline
\end{tabular}

Model 1, calendar year and graft failure; model 2, model $1+$ age at time of transplant, sex, race, BMI, cause of ESRD, and modality duration and dialysis vintage; model 3 , model $2+$ comorbidities, health care utilization metrics, and prior solid organ transplant status; model 4, model 3 + transplant characteristics. Graft failure was treated as a time-varying covariate. All models were stratified by incidence year categories (2005-2006, 2007-2008, 2009-2010, and 2011-2012). CI, confidence interval; HR, hazard ratio. * Subdistribution hazard ratio treats death as a competing event; causespecific hazard ratio treats death as a censoring event.

was associated with a $36 \%(95 \%$ CI: $13 \%-64 \%)$ increase in the sub-distribution hazard of de novo HF in those who were event free or died. However, there was no difference in the sub-distribution hazard between patients treated with hemodialysis versus peritoneal dialysis in other strata of pretransplant BMI below $30 \mathrm{~kg} / \mathrm{m}^{2}$. We also found that among patients with pretransplant diabetes mellitus, hemodialysis (vs. peritoneal dialysis) treatment was associated with a $38 \%$ (95\% CI: $16 \%-65 \%)$ increase in the sub-distribution hazard of de novo HF in those who were event free or died. However, there was no significant difference observed in the sub-distribution hazard of de novo HF in patients without diabetes mellitus treated with hemodialysis versus peritoneal dialysis. Full results for the interaction analysis are shown in Figure 3.

\section{Discussion}

In this study of US patients with ESKD on dialysis who received a first kidney-only transplant, we found that pretransplant treatment with hemodialysis (vs. peritoneal dialysis) was associated with de novo posttransplant HF. After adjustment for numerous potential confounders, those patients treated with hemodialysis before undergoing transplant had an almost $20 \%$ higher risk of being diagnosed with HF in the 3 years after transplant compared to those patients who were treated with peritoneal dialysis. The increased risk of posttransplant HF associated with receipt of pretransplant hemodialysis therapy was greater in those patients with a high BMI and in those with pre-existing diabetes mellitus.

Chronic cardiac volume and pressure overload may complicate both peritoneal dialysis and hemodialysis therapies and can result in the development of left ventricular hypertrophy, which is a precursor of both diastolic and systolic cardiac dysfunction. Both modalities also have distinct (modality specific) features that are relevant to the development of structural cardiac disease and warrant discussion. 


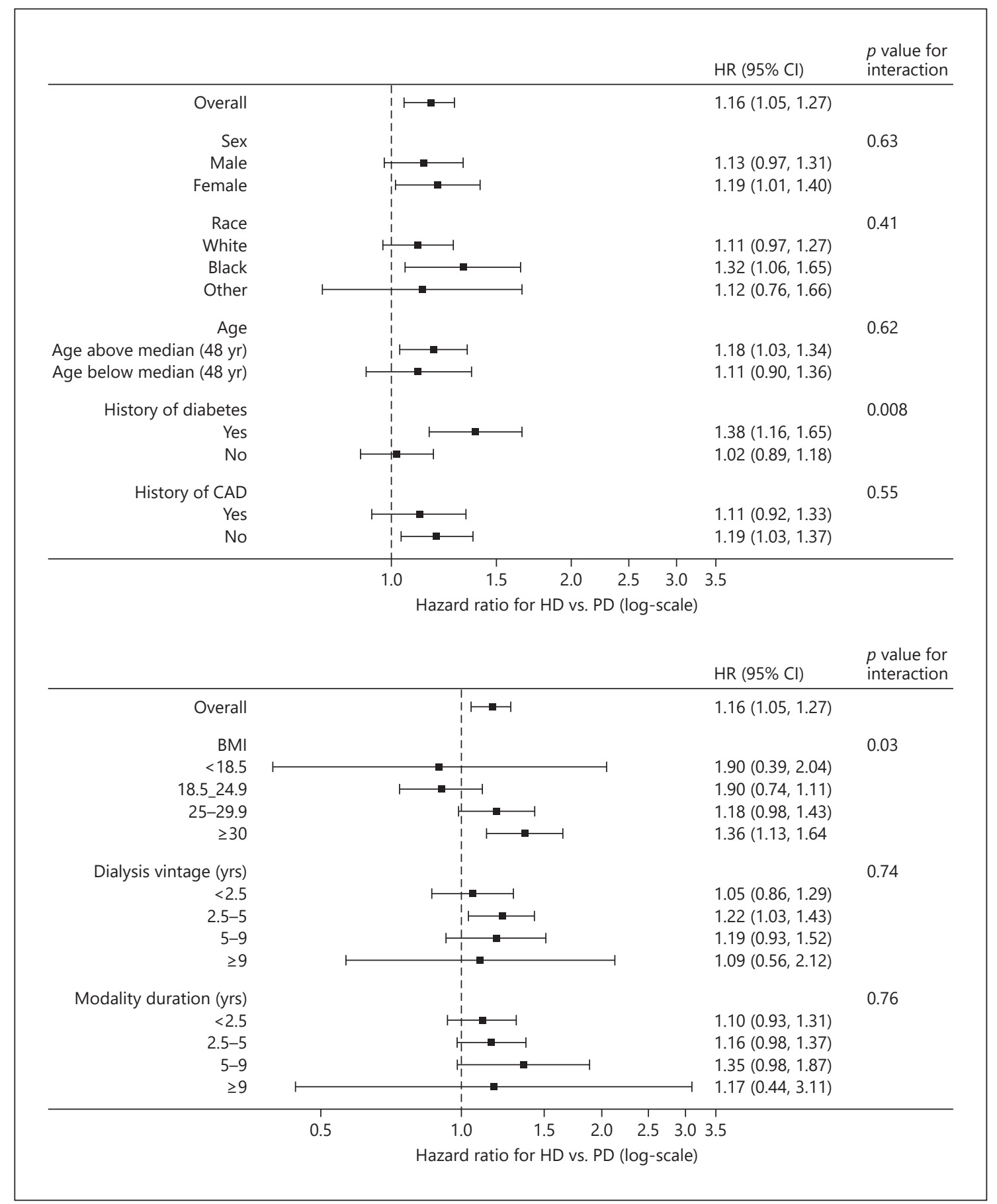

Fig. 3. Test for interactions between selected patient characteristics, pretransplant dialysis modality, and the risk of de novo posttransplant HF. PD, peritoneal dialysis; HD, hemodialysis; HF, heart failure.

Hemodialysis is associated with large, intermittent, and "unphysiological" volume shifts. Large interdialytic weight gains have been shown to be associated with the development of left ventricular hypertrophy while more frequent hemodialysis (compared to standard frequency) appears to protect against the development of left ventricular hypertrophy [15-17]. Hemodialysis-induced myocardial stunning/ischemia is a now well-described 
phenomenon which also likely results in incremental myocardial injury [18]. The use of arteriovenous shunts for hemodialysis access results in an obligate increase in cardiac output and the development of left ventricular hypertrophy. In patients with high-flow arteriovenous fistulas, high output cardiac failure may sometimes ensue. Some (although not all) observational studies show that arteriovenous fistula ligation is associated with favorable left ventricular structural and functional changes $[12,19-21]$; a randomized trial in 64 patients found that arteriovenous fistula ligation caused significant reductions in left ventricular mass and size [22].

Peritoneal dialysis which offers smooth and continuous volume removal should better mimic normal physiology. However, volume control with peritoneal dialysis may be imperfect $[23,24]$. Peritoneal dialysis may also predispose to an unfavorable metabolic and inflammatory milieu which may contribute adversely to cardiac pathophysiology [25]. Sparse data comparing left ventricular hypertrophy prevalence in peritoneal dialysis versus hemodialysis patients yield conflicting results [26, 27]. In patients with existing HF and ESKD, peritoneal dialysis is often favored over hemodialysis as being potentially better hemodynamically tolerated; however, this practice is not supported by the results of a large (albeit retrospective and potentially confounded) study [28].

Our study has several strengths. Our cohort is large and from a relatively recent era. By using the USRDS and linked Medicare fee-for-service claims data, we are able to ascertain richly detailed patient demographic and clinical characteristics. However, as with all retrospective claims-based studies, residual confounding through either incorrectly or uncollected covariates is a possibility. We do not have access to cardiac investigations, dialysis prescriptions, medication history, or laboratory results including posttransplant glomerular filtration rate. We also do not have information regarding the type of pretransplant hemodialysis access.

Posttransplant HF is an important problem and is associated with reduced graft and patient survival [5]. Minimizing cardiac stress and injury should be an important focus of pretransplant management in patients with chronic kidney disease. Ideally, all patients with ESKD should undergo pre-emptive kidney transplant, which is associated with the best posttransplant outcomes $[29,30]$. However, long deceased donor waiting times and insufficient numbers of living donors mean that pre-emptive kidney transplant is not an option for the majority of Americans with ESKD. Therefore, identifying modifiable dialysis-related risk factors for the development of post- transplant heart disease that may help to extend the lives of kidney transplant recipients is imperative. Our findings suggest that further study regarding the impact of pretransplant dialysis modality on posttransplant cardiac function is warranted.

\section{Acknowledgments}

The data reported here have been supplied by the USRDS. The interpretation and reporting of these data are the responsibility of the authors and in no way should be seen as an official policy or interpretation of the US government. None of the authors report a significant financial interest regarding the topic of this study. The results presented in this study have not been published previously in whole or part, except in abstract format.

\section{Statement of Ethics}

This is a retrospective cohort study, and the Internal Review Boards at the Stanford University School of Medicine and Baylor College of Medicine approved the study.

\section{Conflict of Interest Statement}

The authors declare no conflicts of interest.

\section{Funding Sources}

This work was supported by a Mentored Clinical and Population Research Program Grant from the American Heart Association Western States Affiliate (to Dr. Lenihan) and Grant R01DK095024 from the National Institute of Diabetes, Digestive, and Kidney Diseases.

\section{Author Contributions}

C.L. was involved in conception of the work, analysis and interpretation of data, drafting the work, and final approval of the version to be published and agreed to be accountable for all aspects of the work. S.L. was involved in analysis and interpretation of data, revising it critically, and final approval of the version to be published and agreed to be accountable for all aspects of the work. M.A. was involved in analysis and interpretation of data, revising it critically, and final approval of the version to be published and agreed to be accountable for all aspects of the work. C.W. was involved in analysis and interpretation of data, revising it critically, and final approval of the version to be published and agreed to be accountable for all aspects of the work. M.M.R. was involved in analysis and interpretation of data, revising it critically, and final approval of the version to be published and agreed to be accountable for all aspects of the work. W.W. was involved 
in conception of the work; acquisition, analysis, and interpretation of data; revising it critically; and final approval of the version to be published and agreed to be accountable for all aspects of the work.

\section{Data Availability Statement}

Data reported in this study were supplied by the United States Renal Data System (USRDS) - https://www.usrds.org/.

\section{References}

1 Wolfe RA, Ashby VB, Milford EL, Ojo AO, Ettenger RE, Agodoa LY, et al. Comparison of mortality in all patients on dialysis, patients on dialysis awaiting transplantation, and recipients of a first cadaveric transplant. N Engl J Med. 1999;341:1725-30.

2 Laupacis A, Keown P, Pus N, Krueger H, Ferguson $\mathrm{B}$, Wong $\mathrm{C}$, et al. A study of the quality of life and cost-utility of renal transplantation. Kidney Int. 1996;50:235-42.

3 van Walraven C, Manuel DG, Knoll G. Survival trends in ESRD patients compared with the general population in the United States. Am J Kidney Dis. 2014;63:491-9.

4 Collins AJ, Foley RN, Herzog C, Chavers B, Gilbertson D, Herzog C, et al. US renal data system 2012 annual data report. Am J Kidney Dis. 2014;61:A7.

5 Lentine KL, Schnitzler MA, Abbott KC, Li L, Burroughs TE, Irish W, et al. De novo congestive heart failure after kidney transplantation: a common condition with poor prognostic implications. Am J Kidney Dis. 2005;46:72033.

6 Jablonski KL, Chonchol M. Vascular calcification in end-stage renal disease. Hemodial Int. 2013;17(Suppl 1):S17-21.

7 Levin A, Thompson CR, Ethier J, Carlisle EJ, Tobe S, Mendelssohn D, et al. Left ventricular mass index increase in early renal disease: impact of decline in hemoglobin. Am J Kidney Dis. 1999;34:125-34.

8 Gupta J, Dominic EA, Fink JC, Ojo AO, Barrows IR, Reilly MP, et al. Association between inflammation and cardiac geometry in chronic kidney disease: findings from the CRIC study. PLoS One. 2015;10:e0124772.

9 Patel RK, Oliver S, Mark PB, Powell JR, McQuarrie EP, Traynor JP, et al. Determinants of left ventricular mass and hypertrophy in hemodialysis patients assessed by cardiac magnetic resonance imaging. Clin $\mathrm{J}$ Am Soc Nephrol. 2009;4:1477-83.

10 Faul C, Amaral AP, Oskouei B, Hu MC, Sloan A, Isakova T, et al. FGF23 induces left ventricular hypertrophy. J Clin Invest. 2011;121: 4393-408.
11 Foley RN, Parfrey PS, Harnett JD, Kent GM, Murray DC, Barré PE. The prognostic importance of left ventricular geometry in uremic cardiomyopathy. J Am Soc Nephrol. 1995;5: 2024-31.

12 Movilli E, Viola BF, Brunori G, Gaggia P, Camerini C, Zubani R, et al. Long-term effects of arteriovenous fistula closure on echocardiographic functional and structural findings in hemodialysis patients: a prospective study. Am J Kidney Dis. 2010;55:682-9.

13 Ruan PK, Gray RJ. Analyses of cumulative incidence functions via non-parametric multiple imputation. Stat Med. 2008;27:5709-24.

14 Allignol A. kmi: Kaplan-Meier multiple imputation for the analysis of cumulative incidence functions in the competing risks setting. R package version 0.5.3; 2017.

15 Paglialonga F, Consolo S, Galli MA, Testa S, Edefonti A. Interdialytic weight gain in oligoanuric children and adolescents on chronic hemodialysis. Pediatr Nephrol. 2015;30:9991005.

16 Ayus JC, Mizani MR, Achinger SG, Thadhani $\mathrm{R}$, Go AS, Lee S. Effects of short daily versus conventional hemodialysis on left ventricular hypertrophy and inflammatory markers: a prospective, controlled study. J Am Soc Nephrol. 2005;16:2778-88.

17 Chan CT, Greene T, Chertow GM, Kliger AS, Stokes JB, Beck GJ, et al. Determinants of left ventricular mass in patients on hemodialysis: Frequent Hemodialysis Network (FHN) trials. Circ Cardiovasc Imaging. 2012;5:251-61.

18 Burton JO, Jefferies HJ, Selby NM, McIntyre CW. Hemodialysis-induced cardiac injury: determinants and associated outcomes. Clin J Am Soc Nephrol. 2009;4:914-20.

19 Unger P, Velez-Roa S, Wissing KM, Hoang $\mathrm{AD}$, van de Borne P. Regression of left ventricular hypertrophy after arteriovenous fistula closure in renal transplant recipients: a long-term follow-up. Am J Transplant. 2004; 4:2038-44.

20 Unger P, Wissing KM. Arteriovenous fistula after renal transplantation: utility, futility or threat? Nephrol Dial Transplant. 2006;21: 254-7.
21 De Lima JJ, Vieira ML, Molnar LJ, Medeiros CJ, Ianhez LE, Krieger EM. Cardiac effects of persistent hemodialysis arteriovenous access in recipients of renal allograft. Cardiology. 1999;92:236-9.

22 Rao NN, Stokes MB, Rajwani A, Ullah S, Williams K, King D, et al. Effects of Arteriovenous fistula ligation on cardiac structure and function in kidney transplant recipients. Circulation. 2019;139:2809-18.

23 Koc M, Toprak A, Tezcan H, Bihorac A, Akoglu E, Ozener IC. Uncontrolled hypertension due to volume overload contributes to higher left ventricular mass index in CAPD patients. Nephrol Dial Transplant. 2002;17:1661-6.

24 Wang MC, Tseng CC, Tsai WC, Huang JJ. Blood pressure and left ventricular hypertrophy in patients on different peritoneal dialysis regimens. Perit Dial Int. 2001;21:36-42.

25 Wu CK, Huang YT, Lin HH, Yang CY, Lien YC, Lee JK, et al. Dissecting the mechanisms of left ventricular diastolic dysfunction and inflammation in peritoneal dialysis patients. PLoS One. 2013;8:e62722.

26 Lai S, Molfino A, Russo GE, Testorio M, Galani A, Innico G, et al. Cardiac, inflammatory and metabolic parameters: hemodialysis versus peritoneal dialysis. Cardiorenal Med. 2015;5:20-30.

27 Tian JP, Wang T, Wang H, Cheng LT, Tian XK, Lindholm B, et al. The prevalence of left ventricular hypertrophy in Chinese hemodialysis patients is higher than that in peritoneal dialysis patients. Ren Fail. 2008;30:391400 .

28 Stack AG, Molony DA, Rahman NS, Dosekun A, Murthy B. Impact of dialysis modality on survival of new ESRD patients with congestive heart failure in the United States. Kidney Int. 2003;64:1071-9.

29 Mange KC, Joffe MM, Feldman HI. Effect of the use or nonuse of long-term dialysis on the subsequent survival of renal transplants from living donors. N Engl J Med. 2001;344:72631.

30 Davis CL. Preemptive transplantation and the transplant first initiative. Curr Opin Nephrol Hypertens. 2010;19:592-7. 